\title{
Synthesis of Some Pyridazinylacetic Acid Derivatives as a Novel Class of Monoamine Oxidase-A Inhibitors ${ }^{1)}$
}

\author{
Sherine Nabil Khatтaв, ${ }^{a}$ Adnan Ahmed Bekhit,${ }^{b}$ Ayman El-Faham,${ }^{a}$ Abdel Moneim El Massry, ${ }^{a}$ \\ and Adel AmER *,a \\ ${ }^{a}$ Department of Chemistry, Faculty of Science, University of Alexandria; Alexandria 21321, Egypt: and ${ }^{b}$ Department of \\ Pharmaceutical Chemistry, Faculty of Pharmacy, University of Alexandria; Alexandria 21521, Egypt. \\ Received May 3, 2008; accepted August 23, 2008; published online September 25, 2008
}

\begin{abstract}
A series of new pyridazinylacetic acid derivatives were synthesized and have been investigated for their ability to inhibit the activity of the $A$ and $B$ isoforms of monoamine oxidase (MAO). All compounds were found to be more selective to the MAO-A isoform with compound 5d having the highest SI values. Computational study performed with a docking technique indicated the potential of these compounds in pyridazine-based MAO-A inhibitor drug development.
\end{abstract}

Key words monoamine oxidase inhibitor; pyridazine; acetic acid; amino acid

Monoamic oxidase (MAO) (EC 1.4.3.4) is an integral flavin-containing enzyme of the outer mitochondrial membrane which is responsible for regulation and metabolism of major monoamine neurotransmitters such as serotonin $(5-\mathrm{OH}$ tryptamine), noradrenaline and dopamine. It is also involved in the biodegradation of exogenic amines such as benzylamine, tyramine, 1-methyl-4-phenyl-1,2,3,6-tetrahydropyridine (MPTT), 1-methyl-4-phenyl pyridium $\left(\mathrm{MPP}^{+}\right)$, and a Parkinsonian-producing neurotoxin. ${ }^{2}$ It is found in two different isoforms designated as MAO-A and MAO-B which are encoded by two different genes ${ }^{3)}$ and distinguished by different substrate specificities and sensitivities to the selective inhibitors. ${ }^{4}$ )

The new generation of MAO inhibitors is characterized by their relative specificities for the MAO subtypes and in some cases by the reversibility of their actions. In spite of considerable progress in our understanding of the interactions of the two enzyme forms with their preferred substrates and inhibitors, no general rules are yet available for the rational design of potent and selective inhibitors of MAO. This is partly due to the fact that the mechanism of interaction of several new drugs with MAOs has not been fully characterized. Therefore, the discovery of several selective MAO inhibitors has relied on serendipity. Preferential MAO-A inhibitors have been recognized as therapeutically useful antidepressants ${ }^{5)}$, while MAO-B inhibitors have been found to be beneficial in the treatment of Parkinson's disease and Alzheimer's disease. ${ }^{6,7)}$

Recently, we have demonstrated a series of 3-benzyl-2substituted quinoxalines as selective MAO-A inhibitors bearing substituted amino or hydrazino functionalities at position 2 (e.g. 2-morpholinoethylamino, 2-hydroxyethylamino, hydrazino, etc). ${ }^{8)}$ The rational design of these compounds was based on a hybrid structure of known inhibitors. The aim of the present study was to design MAO-A inhibitors while taking into consideration various factors responsible for selectivity against the A isoform, ${ }^{9}{ }^{9}$ namely i) the presence of electron-rich aromatic moieties (e.g. Bazinaprine $\left.{ }^{10)}\right)$, ii) the presence of hydrazido functionality (e.g. Iproniazid ${ }^{11)}$ ), iii) the presence of an ethoxycarbonyl methylene group side chain of an aromatic system (e.g. Eugenol analog ${ }^{12)}$ ); exchanging the ester group with an amido group was also considered, and iv) the benzyl group was retained in the new model compounds in Fig. 1.

The new substituted pyridazine-1-yl acetic acid derivatives $(\mathbf{2}, \mathbf{3}, \mathbf{5})$ were designed as selective monoamine oxidase-A inhibitors. The synthesis and biological evaluation of these compounds were described. As a preliminary result of the biological investigation, compound 5d showed the highest MAO-A selectivity and could be the lead compound for designing more potent and selective MAO-A inhibitors.

First, the 4-benzyl-2H-pyridazin-3-one derivatives 1 were prepared. The reaction of the 4-benzyl-2H-pyridazin-3-one derivatives 1 with bromo ethyl acetate in dimethyl formamide in the presence of potassium iodide and sodium bicarbonate afforded the (5-benzyl-6-oxo-6H-pyridazin-1-yl)-

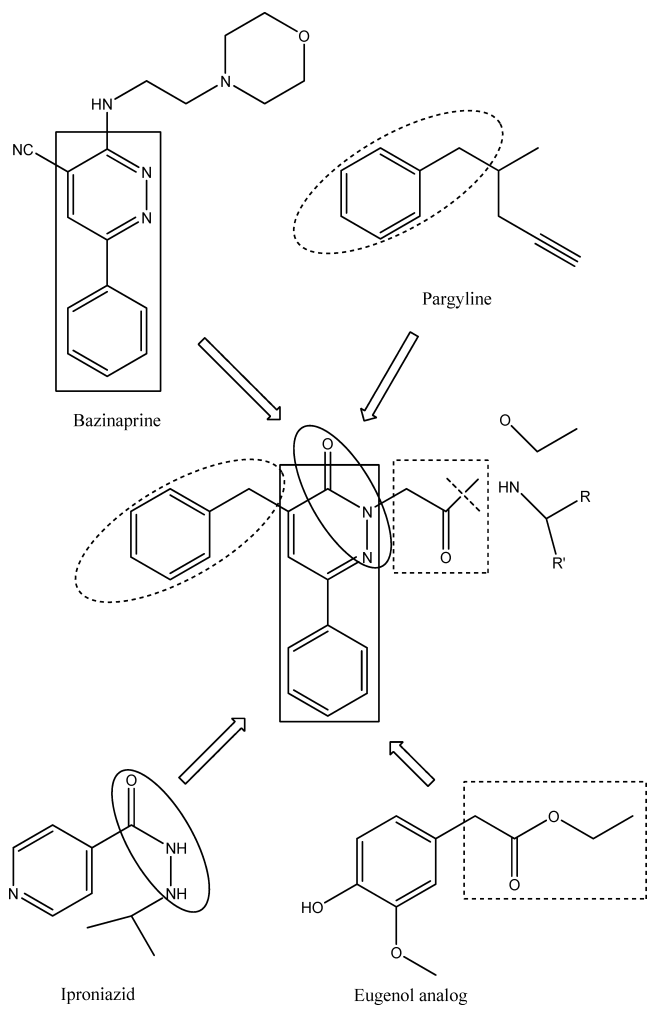

Fig. 1. Planned Modification and Newly Designed MAO-A Inhibitor 


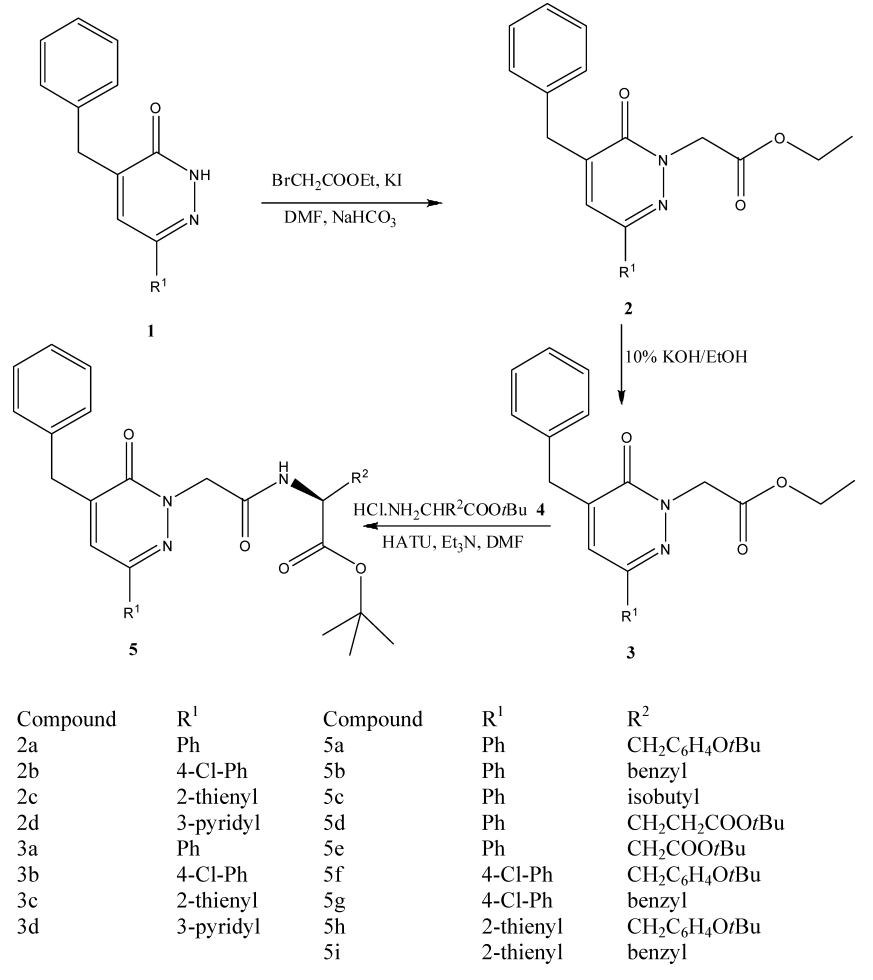

Chart 1

acetic acid ethyl ester and its derivatives $\mathbf{2}$. The esters $\mathbf{2 a}, \mathbf{2 b}$ and $2 \mathbf{c}$ were hydrolysed by alcoholic potassium hydroxide to give the corresponding acids $\mathbf{3 a}, \mathbf{3 b}$ and $\mathbf{3 c}$, while the ester 2d was hydrolyzed by acid hydrolysis by the use of $5 \mathrm{~N} \mathrm{HCl}$. The structures of compounds $\mathbf{2 a}-\mathbf{d}$ and $\mathbf{3 a}-\mathbf{d}$ were established by IR, ${ }^{1} \mathrm{H}-\mathrm{NMR},{ }^{13} \mathrm{C}-\mathrm{NMR}$, and elemental analyses.

The reaction of 2-(5-benzyl-6-oxo-3-phenylpyridazin$1(6 H)$-yl)acetic acid $\mathbf{3 a}$ and its derivatives $\mathbf{3 b}$ and $\mathbf{3 c}$ with different L-amino acid $t$-butyl esters $\mathbf{4} \mathbf{a}-\mathbf{e}$ in dimethylformamide in the presence of triethylamine and HATU as coupling reagent at $0{ }^{\circ} \mathrm{C}$ yielded the derivatives $\mathbf{5}$ in high yield (Chart 1). The structures of compounds 5 were established by IR, ${ }^{1} \mathrm{H}$-NMR, ${ }^{13} \mathrm{C}$-NMR and elemental analyses. The infrared spectra of these compounds showed absorption bands of the ester carbonyl groups at the $1731-1732 \mathrm{~cm}^{-1}$ range, of the amide carbonyl groups are approximately at 1659 $\mathrm{cm}^{-1}$ and at $1690 \mathrm{~cm}^{-1}$ (the first one is the one conjugated with the adjacent double bond), and of the $\mathrm{N}-\mathrm{H}$ at the $3308-3313 \mathrm{~cm}^{-1}$ range. In addition, ${ }^{1} \mathrm{H}-\mathrm{NMR}$ spectra showed only one exchangeable signal for the $\mathrm{NH}$ group. For further structure elucidation, the DEPT technique was performed for compound $\mathbf{5 c}$ as a prototype.

The newly synthesized compounds $\mathbf{2}, \mathbf{3}, \mathbf{5}$ were evaluated for their MAO-A inhibitory activity in vitro by the method described by Undenfriend et al. ${ }^{13}$ by determination of the MAO-A activity of rat liver mitochondria ${ }^{14)}$ (Table 1).

Furthermore, the synthesized compounds 2, 3, 5 were tested to determine their activity toward MAO-A and MAO$\mathrm{B}$ selectivity in the presence of a specific substrate, ${ }^{15)}$ serotonin or benzylamine respectively. Bovine brain mitochondria were isolated according to Basford. ${ }^{15)}$ The compounds 2 , $\mathbf{3}, \mathbf{5}$ were tested to determine their activity toward MAO-A and MAO-B according to the methods of Matsumoto et al. ${ }^{16)}$ and Bradford. ${ }^{17)}$ The MAO-A and MAO-B results are ex-
Table 1. Effect of Some Pyridazinyl Acetic Acid Derivatives 2, 3 and 5 on the Activity of MAO-A and MAO-B

\begin{tabular}{ccccr}
\hline \hline Compound & $\begin{array}{c}\text { \% MAO-A } \\
\text { inhibition }\end{array}$ & MAO-A IC & & MAO-B IC \\
50 & $\begin{array}{r}\text { Selectivity } \\
\text { index (SI) }\end{array}$ \\
\hline 2a & $43.75 \pm 1.22$ & $7.3 \times 10^{-9} \pm 0.04$ & $9.2 \times 10^{-4} \pm 0.55$ & 126027 \\
$\mathbf{2 b}$ & $29.45 \pm 1.98$ & $1.8 \times 10^{-8} \pm 0.09$ & $2.2 \times 10^{-4} \pm 0.06$ & 12222 \\
$\mathbf{2 c}$ & $40.65 \pm 1.94$ & $8.1 \times 10^{-9} \pm 0.02$ & $7.4 \times 10^{-4} \pm 0.21$ & 91358 \\
$\mathbf{2 d}$ & $34.86 \pm 2.47$ & $9.7 \times 10^{-9} \pm 0.02$ & $4.6 \times 10^{-4} \pm 0.18$ & 47422 \\
$\mathbf{3 a}$ & $11.64 \pm 1.56$ & $6.2 \times 10^{-7} \pm 0.04$ & $1.9 \times 10^{-4} \pm 0.08$ & 306 \\
$\mathbf{3 b}$ & $33.70 \pm 3.44$ & $9.8 \times 10^{-9} \pm 0.06$ & $3.6 \times 10^{-5} \pm 0.27$ & 3673 \\
$\mathbf{3 c}$ & $29.84 \pm 4.12$ & $2.3 \times 10^{-8} \pm 0.05$ & $8.2 \times 10^{-4} \pm 0.22$ & 35652 \\
$\mathbf{5 a}$ & $37.95 \pm 1.87$ & $9.4 \times 10^{-9} \pm 0.16$ & $4.7 \times 10^{-5} \pm 0.07$ & 5000 \\
$\mathbf{5 b}$ & $30.96 \pm 3.12$ & $9.7 \times 10^{-9} \pm 0.08$ & $7.1 \times 10^{-5} \pm 0.36$ & 7319 \\
$\mathbf{5 c}$ & $27.12 \pm 2.54$ & $2.9 \times 10^{-8} \pm 0.14$ & $1.4 \times 10^{-4} \pm 0.18$ & 4827 \\
$\mathbf{5 d}$ & $57.65 \pm 1.74$ & $5.4 \times 10^{-9} \pm 0.02$ & $8.1 \times 10^{-4} \pm 0.43$ & 150000 \\
$\mathbf{5 e}$ & $30.98 \pm 2.84$ & $9.6 \times 10^{-9} \pm 0.12$ & $2.7 \times 10^{-4} \pm 0.28$ & 28125 \\
$\mathbf{5 f}$ & $38.73 \pm 1.82$ & $1.4 \times 10^{-8} \pm 0.16$ & $2.3 \times 10^{-4} \pm 0.14$ & 16428 \\
$\mathbf{5 g}$ & $32.92 \pm 2.88$ & $2.8 \times 10^{-8} \pm 0.01$ & $2.6 \times 10^{-4} \pm 0.52$ & 9285 \\
$\mathbf{5 h}$ & $30.99 \pm 2.68$ & $2.6 \times 10^{-8} \pm 0.25$ & $7.5 \times 10^{-4} \pm 0.28$ & 28846 \\
$\mathbf{5 i}$ & $36.42 \pm 2.88$ & $1.7 \times 10^{-8} \pm 0.13$ & $4.3 \times 10^{-4} \pm 0.33$ & 25294 \\
\hline
\end{tabular}

a) $\mathrm{SI}=\mathrm{MAO}-\mathrm{B} \mathrm{IC}_{50} / \mathrm{MAO}-\mathrm{A} \mathrm{IC} \mathrm{IC}_{50} *$ The results were expressed as mean \pm S.E.M Data were analyzed by one way of variance. Student's $t$ test for unpaired observations was used. $p$ value $=<0.001$ and was significant. Number of experiments was 6 .

pressed as $\mathrm{IC}_{50}$ (Table 1). The selectivity index is also given in Table 1 . The results revealed that compound $\mathbf{5 d}$ showed MAO-A inhibition greater than that of pargyline $\mathrm{HCl}(\%$ MAO-A inhibition $=39.14 \pm 1.32$ ), while compounds 2a, 2c, $\mathbf{2 d}, \mathbf{3 b}, \mathbf{5 a}, \mathbf{5 f}$ and $\mathbf{5 i}$ exhibited inhibition activity more or less comparable to the standard, pargyline $\mathrm{HCl}$. These compounds showed higher MAO-A inhibitory activity than MAO-B inhibitory activity. Thus, compound 5d appears to be the most selective MAO-A inhibitor.

In an attempt to understand the reason for the observed MAO-A inhibitory activity, a molecular modelling study of compounds 2a, 3a and 5d, as well as conformational alignment studies were performed in order to rationalize the obtained biological results. Molecular docking studies can further assist in understanding in detail the various interactions between the ligands and enzyme active sites in detail.

Molecular operating environment (MOE) $)^{18)}$ docking studies of the inhibitors were performed using human MAO-A crystal structure (PDB ID: 2BXR).

Docking of compound 2a into the MAO-A active site revealed that several molecular interactions were considered to be responsible for the observed affinity: Hydrophobic interactions were observed with each of the following residues: PHE208, ILE335, LEU337, MET350. Docking of the corresponding acid derivative 3a showed less hydrophobic interactions with the backbone of MAO-A enzyme with the following residues: VAL93, LEU97, PHE208. On the other hand, docking of compound 5d into the MAO-A active site (Fig. 2) showed that the molecular interactions responsible for the observed affinity are: i) two hydrogen bond interactions with the following residue: SER209. ii) Hydrophobic interactions with the following residues: PHE352, Va193, Leu97, ILE180, PHE208, ILE325, ILE335, ILE337, TRP441. These results showed that compound 5d bound to the MAO-A active site with a position and orientation that were superior of those of 2a and 3a.

It is worth to mentioning that the test compounds were further tested for their oral acute toxicity in male mice using a literature method. ${ }^{18,19)}$ The results revealed that the test com- 


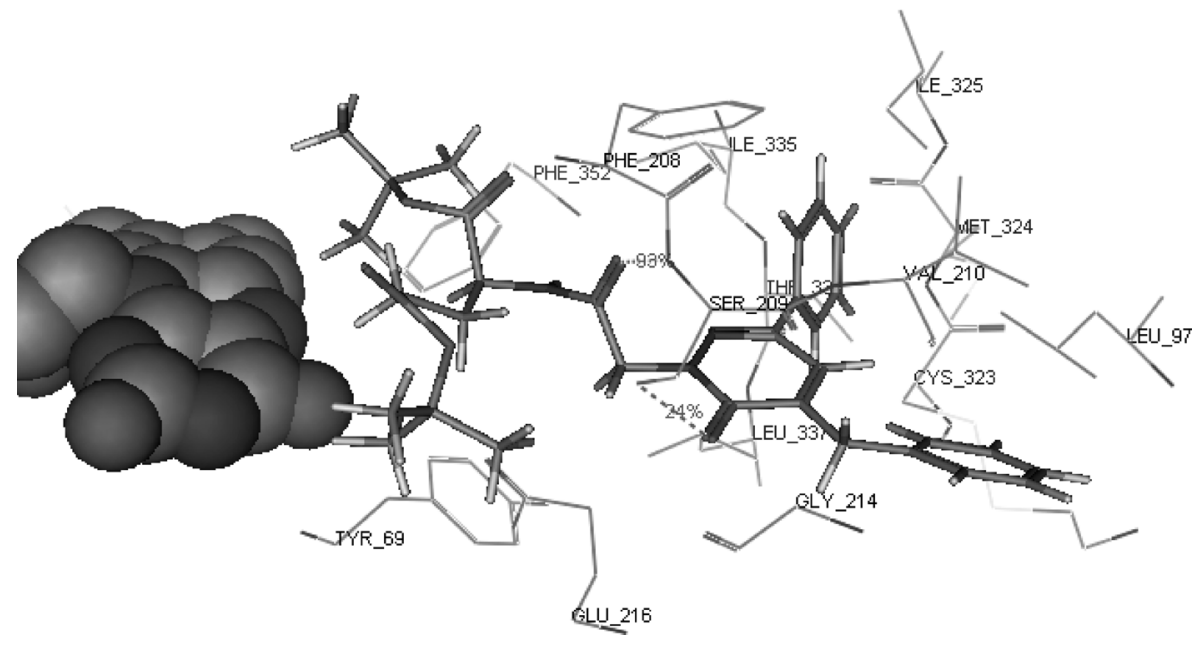

Fig. 2. 3D View from a Molecular Modelling Study, of Minimum-Energy Structure of the Complex of 5d (Stick) Docked in the Active Site of MAO-A (PDB ID: 2BXR)

Dashed lines depict hydrogen bond interactions. The space filling model at the left side represents FAD coenzyme. Viewed using Molecular Operating Environment (MOE) module.

pounds proved to be non-toxic and well tolerated by the experimental animals up to $250 \mathrm{mg} / \mathrm{kg}$, although no mortality was recorded at $500 \mathrm{mg} / \mathrm{kg}$. Furthermore, these compounds were evaluated for their toxicity when administered via the parenteral route. ${ }^{20)}$ The results showed that all of the test compounds were non-toxic up to $125 \mathrm{mg} / \mathrm{kg}$.

From the above mentioned data and its non toxic behaviour, ${ }^{18-20)}$ 5d may provide a starting point for the design of unique compounds with high affinity and selectivity for MAO-A.

\section{Experimental}

Chemistry Melting points were determined with a Mel-Temp apparatus and are uncorrected. Magnetic resonance spectra $\left({ }^{1} \mathrm{H}-\mathrm{NMR}\right.$ and ${ }^{13} \mathrm{C}-\mathrm{NMR}$ spectra) were recorded on a JEOL $500 \mathrm{MHz}$ spectrometer with chemical shift values reported in $\delta$ units (part per million) relative to an internal standard (tetramethylsilane). Splitting patterns were designated as follows: s: singlet; d: doublet; t: triplet; q: quartet, m: multiplet. Infrared (IR) data were obtained on a Perkin-Elmer 1600 series Fourier transform instrument as $\mathrm{KBr}$ pellets. Mass spectra were recorded on a QP 1000EX GC/MS (Shimadzu Corp.). Elemental analyses were performed on a Perkin-Elmer 2400 elemental analyzer, and the values were within $\pm 0.3 \%$ of the theoretical values. The reactions were followed-up and the purity of the compounds was checked by TLC on silica gel-protected aluminium sheets (Type 60 GF254, Merck) and the spots were detected by exposure to a UV-lamp at $\lambda 254 \mathrm{~nm}$ for several seconds. The compounds were named using Chem. Draw Ultra version 6 (Cambridgesoft Corporation). The following abbreviations are used throughout the manuscript: HATU, $N$-[(dimethylamino)- $1 H-1,2,3-$ triazolo[4,5- $b$ ]pyridin-1-ylmethylene]- $N$-methylmethanaminium hexafluorophosphate $N$-oxide, $\mathrm{Et}_{3} \mathrm{~N}$, triethylamine, and DMF, $N, N$-dimethylformamide were used through out the manuscript.

All molecular modeling studies were carried out on an Intel Pentium 1.6 GHz processor, $512 \mathrm{MB}$ memory with Windows XP operating system using Molecular Operating Environment (MOE 2005.06; Chemical Computing Group, Montréal, Canada) as the computational software. All minimizations were performed with MOE until a RMSD gradient of 0.05 kcal mol ${ }^{-1} \AA^{-1}$ with MMFF94X forcefield and the partial charges were automatically calculated. Docking and molecular modeling calculations were carried out at the Department of Pharmaceutical and Medicinal Chemistry, Faculty of Pharmacy, Assiut University, Assiut, Egypt.

General Procedure for the Preparation of 4-Benzylpyridazin-3(2H)one Derivatives (1a-d) A solution of 2-benzyl-2-hydroxy-4-oxo-4 arylbutanoic acid $(2 \mathrm{mmol})$ in ethanol $(20 \mathrm{ml})$ was refluxed with $90 \%$ hydrazine hydrate $(2 \mathrm{ml})$ for $3 \mathrm{~h}$. The product which was separated out on concentration was collected by filtration, dried, and recrystallized from ethanol.

4-Benzyl-6-phenylpyridazin-3(2H)-one (1a): This compound was ob- tained as colorless crystals, $0.36 \mathrm{~g}(68.6 \%), \mathrm{mp} 178-180^{\circ} \mathrm{C}$ (lit. mp $179-$ $\left.181^{\circ} \mathrm{C}\right) .{ }^{21,22)}$

4-Benzyl-6-(4-chlorophenyl)pyridazin-3(2H)-one (1b): This compound was obtained as colorless crystals, $0.47 \mathrm{~g}(79.2 \%), \mathrm{mp} 218-220^{\circ} \mathrm{C}$. Anal. Calcd for $\mathrm{C}_{17} \mathrm{H}_{13} \mathrm{ClN}_{2} \mathrm{O}: \mathrm{C}, 68.81 ; \mathrm{H}, 4.41 ; \mathrm{N}, 9.44$. Found: $\mathrm{C}, 68.81 ; \mathrm{H}$, $4.12 ; \mathrm{N}, 9.56$

4-Benzyl-6-(thiophen-2-yl)pyridazin-3(2H)-one (1c): This compound was obtained as colorless crystals, $0.39 \mathrm{~g}(72.7 \%), \mathrm{mp} 135-137^{\circ} \mathrm{C}$. Anal. Calcd for $\mathrm{C}_{15} \mathrm{H}_{12} \mathrm{~N}_{2} \mathrm{OS}: \mathrm{C}, 67.14 ; \mathrm{H}, 4.51 ; \mathrm{N}, 10.44$. Found: $\mathrm{C}, 67.08 ; \mathrm{H}, 4.51 ; \mathrm{N}$, 10.56

4-Benzyl-6-(pyridin-3-yl)pyridazin-3(2H)-one (1d): This compound was obtained as colorless crystals, $0.47 \mathrm{~g}(89.3 \%), \mathrm{mp} 214-216^{\circ} \mathrm{C}$. Anal. Calcd for $\mathrm{C}_{16} \mathrm{H}_{13} \mathrm{~N}_{3} \mathrm{O}: \mathrm{C}, 72.99 ; \mathrm{H}, 4.98 ; \mathrm{N}, 15.96$. Found: $\mathrm{C}, 72.77 ; \mathrm{H}, 5.02 ; \mathrm{N}$, 15.66

General Procedure for the Preparation of Compounds (2a-d) 4 Benzylpyridazin-3 $(2 \mathrm{H})$-one derivatives $1(2 \mathrm{mmol})$ were dissolved in dry DMF (5 ml), NaHCO $3(3.02 \mathrm{~g}, 36 \mathrm{mmol})$ and $\mathrm{KI}(0.432 \mathrm{~g}, 2.6 \mathrm{mmol})$ and the mixture was stirred at room temperature for $15 \mathrm{~min}$. Excess of ethyl bromoacetate $(1.33 \mathrm{~g}, 8 \mathrm{mmol})$ was then added and the reaction mixture was kept stirring at room temperature for $84 \mathrm{~h}$. DMF was removed under reduced pressure. The residue was suspended in water $(150 \mathrm{ml})$ and extracted with methylene chloride $(100 \mathrm{ml})$. The organic layer was dried over anhydrous sodium sulphate, filtered and the solvent was removed under reduced pressure. The crude product was crystallized from ethanol.

Ethyl 2-(5-Benzyl-6-oxo-3-phenylpyridazin-1 $(6 H)$-yl)acetate (2a): This compound was obtained as colorless crystals, $0.64 \mathrm{~g}(92.0 \%), \mathrm{mp} 92-93^{\circ} \mathrm{C}$ (lit. $\left.\mathrm{mp} 96^{\circ} \mathrm{C}\right)^{22)}$; $\mathrm{IR}(\mathrm{KBr}) \mathrm{cm}^{-1}: 1760(\mathrm{C}=\mathrm{O}$ ester $), 1651(\mathrm{C}=\mathrm{O}$ amide $)$; ${ }^{1} \mathrm{H}-\mathrm{NMR}\left(\mathrm{CDCl}_{3}\right) \delta: 1.29\left(\mathrm{t}, 3 \mathrm{H}, \mathrm{CH}_{3}, J=6.9 \mathrm{~Hz}\right), 3.99\left(\mathrm{~s}, 2 \mathrm{H}, \mathrm{CH}_{2}\right), 4.26$ $\left(\mathrm{q}, 2 \mathrm{H}, \mathrm{CH}_{2}, J=6.9 \mathrm{~Hz}\right), 4.98\left(\mathrm{~s}, 2 \mathrm{H}, \mathrm{CH}_{2}\right), 7.26-7.42(2 \mathrm{~m}, 9 \mathrm{H}, \mathrm{PyrH}$ ArH), $7.63-7.65(\mathrm{~m}, 2 \mathrm{H}, \mathrm{ArH})$. MS [EI]: $m / e 348\left(\mathrm{M}^{+}, 100 \%\right), 302(22)$, 274 (95), 246 (38), 203 (25), 104 (16), 91 (36), 77 (31). Anal. Calcd for $\mathrm{C}_{21} \mathrm{H}_{20} \mathrm{~N}_{2} \mathrm{O}_{3}: \mathrm{C}, 72.40 ; \mathrm{H}, 5.79 ; \mathrm{N}, 8.04$. Found: $\mathrm{C}, 72.60 ; \mathrm{H}, 5.95 ; \mathrm{N}, 7.88$.

Ethyl 2-(5-Benzyl-3-(4-chlorophenyl)-6-oxopyridazin-1(6H)-yl)acetate (2b): This compound was obtained as colorless crystals, $0.71 \mathrm{~g}(92.9 \%), \mathrm{mp}$ 127-128 ${ }^{\circ} \mathrm{C}$; IR $(\mathrm{KBr}) \mathrm{cm}^{-1}: 1761(\mathrm{C}=\mathrm{O}$ ester $), 1650(\mathrm{C}=\mathrm{O}$ amide $) ;{ }^{1} \mathrm{H}$ NMR $\left(\mathrm{CDCl}_{3}\right) \delta: 1.29\left(\mathrm{t}, 3 \mathrm{H}, \mathrm{CH}_{3}, J=6.9 \mathrm{~Hz}\right), 3.98\left(\mathrm{~s}, 2 \mathrm{H}, \mathrm{CH}_{2}\right), 4.26(\mathrm{q}$, $\left.2 \mathrm{H}, \mathrm{CH}_{2}, J=6.9 \mathrm{~Hz}\right), 4.98\left(\mathrm{~s}, 2 \mathrm{H}, \mathrm{CH}_{2}\right), 7.22(\mathrm{~s}, 1 \mathrm{H}, \mathrm{PyrH}), 7.26,7.35,7.37$, 7.57 (m, d, m, d, 9H, ArH). MS [EI]: m/e 384, $382\left(\mathrm{M}^{+}, 33,93 \%\right), 354(50)$ 308 (90), 203 (30), 196 (23), 189 (20), 139 (57), 115 (63), 113, (27), 111 (30), 91 (40), 51 (100). Anal. Calcd for $\mathrm{C}_{21} \mathrm{H}_{19} \mathrm{ClN}_{2} \mathrm{O}_{3}: \mathrm{C}, 65.88 ; \mathrm{H}, 5.00 ; \mathrm{N}$, 7.32. Found: C, $66.15 ; \mathrm{H}, 5.22 ; \mathrm{N}, 7.04$

Ethyl 2-(5-Benzyl-6-oxo-3-(thiophen-2-yl)pyridazin-1(6H)-yl)acetate (2c): This compound was obtained as colorless crystals, $0.65 \mathrm{~g}(91.8 \%), \mathrm{mp}$ 113-114 ${ }^{\circ} \mathrm{C}$; IR (KBr) cm ${ }^{-1}: 1761$ ( $\mathrm{C}=\mathrm{O}$ ester), $1652\left(\mathrm{C}=\mathrm{O}\right.$ amide); ${ }^{1} \mathrm{H}-$ NMR $\left(\mathrm{CDCl}_{3}\right) \delta: 1.29\left(\mathrm{t}, 3 \mathrm{H}, \mathrm{CH}_{3}, J=6.9 \mathrm{~Hz}\right), 3.96\left(\mathrm{~s}, 2 \mathrm{H}, \mathrm{CH}_{2}\right), 4.25(\mathrm{q}$, $\left.2 \mathrm{H}, \mathrm{CH}_{2}, J=6.9 \mathrm{~Hz}\right), 4.92\left(\mathrm{~s}, 2 \mathrm{H}, \mathrm{CH}_{2}\right), 7.00(\mathrm{dd}, 1 \mathrm{H}, \mathrm{ThH}-4, J=5.4$ $3.9 \mathrm{~Hz}), 7.18(\mathrm{~s}, 1 \mathrm{H}, \mathrm{PyrH}), 7.19(\mathrm{~d}, 1 \mathrm{H}, \mathrm{ThH}-3, J=3.9 \mathrm{~Hz}), 7.26-7.37(\mathrm{~m}$, $6 \mathrm{H}, \mathrm{ThH}-5, \mathrm{ArH}) .{ }^{13} \mathrm{C}-\mathrm{NMR}\left(\mathrm{CDCl}_{3}-\mathrm{HMQC}\right) \delta: 14.23,36.17,54.02,61.81$, 
$125.68,126.95,127.11,127.76,127.91,128.96,129.57,136.94,139.24$, 141.11, 143.63, 160.22, 167.50. MS [EI]: m/e 356, 355, $354\left(\mathrm{M}^{+}, 46,36\right.$, 100\%), 310 (51), 309 (33), 308 (99), 245 (42),203 (68), 202 (85), 138 (32), 101 (86), 51 (71). Anal. Calcd for $\mathrm{C}_{19} \mathrm{H}_{18} \mathrm{~N}_{2} \mathrm{O}_{3} \mathrm{~S}: \mathrm{C}, 64.39 ; \mathrm{H}, 5.12 ; \mathrm{N}, 7.90$. Found: C, 64.10; H, 4.86; N, 8.17.

Ethyl 2-(5-Benzyl-6-oxo-3-(pyridin-3-yl)pyridazin-1(6H)-yl)acetate (2d): This compound was obtained as colorless crystals, $0.59 \mathrm{~g}(84.5 \%), \mathrm{mp}$ $112-113^{\circ} \mathrm{C}$; IR $(\mathrm{KBr}) \mathrm{cm}^{-1}: 1760(\mathrm{C}=\mathrm{O}$ ester $), 1651(\mathrm{C}=\mathrm{O}$ amide $) \mathrm{cm}^{-1}$; ${ }^{1} \mathrm{H}-\mathrm{NMR}\left(\mathrm{CDCl}_{3}\right) \delta$ : $1.28\left(\mathrm{t}, 3 \mathrm{H}, \mathrm{CH}_{3}, J=6.9 \mathrm{~Hz}\right), 3.99\left(\mathrm{~s}, 2 \mathrm{H}, \mathrm{CH}_{2}\right), 4.25$ (q, $\left.2 \mathrm{H}, \mathrm{CH}_{2}, J=6.9 \mathrm{~Hz}\right), 4.97\left(\mathrm{~s}, 2 \mathrm{H}, \mathrm{CH}_{2}\right), 7.24-7.28$ (m, 2H, PyrH, ArH), $7.31-7.33(\mathrm{~m}, 4 \mathrm{H}, \mathrm{ArH}), 7.72(\mathrm{dt}, 1 \mathrm{H}, \mathrm{PyH}-5, J=7.7,2.3 \mathrm{~Hz}), 8.06(\mathrm{~d}, 1 \mathrm{H}$ PyH-4, $J=7.7$ Hz), 8.09 (br s, $1 \mathrm{H}, \mathrm{PyH}-2$ ), 8.57 (d, 1H, PyH-6, $J=7.7 \mathrm{~Hz}$ ). MS [EI]: m/e $349\left(\mathrm{M}^{+}, 45 \%\right), 275$ (25), 247 (34), 204 (22), 115 (55), 105 (28), 91 (45), 78 (100). Anal. Calcd for $\mathrm{C}_{20} \mathrm{H}_{19} \mathrm{~N}_{3} \mathrm{O}_{3}$ : C, 68.75; H, 5.48; N, 12.03. Found: C, 69.03; H, 5.70; N, 11.84 .

General Procedure for the Preparation of Compounds (3a-c) Ester $2(1 \mathrm{mmol})$ was dissolved in $10 \% \mathrm{KOH}$ in ethanol $(20 \mathrm{ml})$. The reaction mixture was refluxed for $3 \mathrm{~h}$ and then neutralized with $1 \mathrm{~N} \mathrm{HCl}$ solution. The precipitate formed was filtered off and washed with water to give the acid 3 .

2-(5-Benzyl-6-oxo-3-phenylpyridazin-1(6H)-yl)acetic Acid (3a): This compound was obtained as white powder, $0.29 \mathrm{~g}(90.5 \%), \mathrm{mp} 180-181^{\circ} \mathrm{C}$; IR $(\mathrm{KBr}) \mathrm{cm}^{-1}$ : $3087-2565(\mathrm{br}, \mathrm{OH}), 1728(\mathrm{C}=\mathrm{O}$ acid $), 1646(\mathrm{C}=\mathrm{O}$ amide); ${ }^{1} \mathrm{H}-\mathrm{NMR}\left(\mathrm{CDCl}_{3}\right) \delta: 3.98\left(\mathrm{~s}, 2 \mathrm{H}, \mathrm{CH}_{2}\right), 5.02\left(\mathrm{~s}, 2 \mathrm{H}, \mathrm{CH}_{2}\right), 7.23$ $7.39,7.62-7.63(2 \mathrm{~m}, 11 \mathrm{H}, \mathrm{ArH}, \mathrm{PyrH}), 13.3\left(\mathrm{~s}, 1 \mathrm{H}, \mathrm{COOH}, \mathrm{D}_{2} \mathrm{O}\right.$ exchangeable). MS [EI]: m/e $320\left(\mathrm{M}^{+}, 35 \%\right), 274$ (27), 203 (32), 178 (12) 104 (35), 102 (43), 91 (46), 77 (100). Anal. Calcd for $\mathrm{C}_{19} \mathrm{H}_{16} \mathrm{~N}_{2} \mathrm{O}_{3} \cdot \mathrm{H}_{2} \mathrm{O}: \mathrm{C}$, 67.44; H, 5.36; N, 8.28. Found: C, 67.18; H, 5.14; N, 8.53.

2-(5-Benzyl-3-(4-chlorophenyl)-6-oxopyridazin-1(6H)-yl)acetic Acid (3b): This compound was obtained as white powder, $0.32 \mathrm{~g}(90.2 \%), \mathrm{mp}$ $194-195^{\circ} \mathrm{C}$; IR (KBr) cm ${ }^{-1}$ : $3030-2581$ (br, OH), 1729 ( $\mathrm{C}=\mathrm{O}$ acid), $1626(\mathrm{C}=\mathrm{O}$ amide $) ;{ }^{1} \mathrm{H}-\mathrm{NMR}\left(\mathrm{DMSO}-d_{6}\right) \delta: 3.86\left(\mathrm{~s}, 2 \mathrm{H}, \mathrm{CH}_{2}\right), 4.81(\mathrm{~s}, 2 \mathrm{H}$ $\left.\mathrm{CH}_{2}\right), 7.18(\mathrm{t}, 1 \mathrm{H}, \mathrm{ArH}, J=7.6 \mathrm{~Hz}), 7.27(\mathrm{t}, 2 \mathrm{H}, \mathrm{ArH}, J=7.6 \mathrm{~Hz}), 7.31(\mathrm{~d}$ $2 \mathrm{H}, \mathrm{ArH}, J=7.6 \mathrm{~Hz}), 7.53(\mathrm{~d}, 2 \mathrm{H}, \mathrm{ArH}, J=8.4 \mathrm{~Hz}), 7.81$ (d, 2H, ArH $J=8.4 \mathrm{~Hz}$ ), $7.96(\mathrm{~s}, 1 \mathrm{H}, \mathrm{PyrH}), 13.2$ (s, 1H, COOH, $\mathrm{D}_{2} \mathrm{O}$ exchangeable). MS [EI]: $m / e$ 356, $354\left(\mathrm{M}^{+}, 21,82 \%\right), 308$ (17), 280 (100), 252 (51), 178 (24) 115 (61), 113 (5), 111 (13), 110 (30), 91 (67), 77 (27). Anal. Calcd for $\mathrm{C}_{19} \mathrm{H}_{15} \mathrm{ClN}_{2} \mathrm{O}_{3}: \mathrm{C}, 64.32 ; \mathrm{H}, 4.26 ; \mathrm{N}, 7.90$. Found: C, 64.09; H, 3.98; N, 7.76 .

2-(5-Benzyl-6-oxo-3-(thiophen-2-yl)pyridazin-1(6H)-yl)acetic Acid (3c): This compound was obtained as white powder, $0.29 \mathrm{~g}(89.0 \%), \mathrm{mp} 249$ $250{ }^{\circ} \mathrm{C}$; IR $(\mathrm{KBr}) \mathrm{cm}^{-1}: 3076-2576(\mathrm{br}, \mathrm{OH}), 1728(\mathrm{C}=\mathrm{O}$ acid $), 1626$ $\left(\mathrm{C}=\mathrm{O}\right.$ amide); ${ }^{1} \mathrm{H}-\mathrm{NMR}\left(\mathrm{DMSO}-d_{6}\right) \delta: 3.85\left(\mathrm{~s}, 2 \mathrm{H}, \mathrm{CH}_{2}\right), 4.73(\mathrm{~s}, 2 \mathrm{H}$, $\left.\mathrm{CH}_{2}\right), 7.12(\mathrm{dd}, 1 \mathrm{H}, \mathrm{ThH}-4, J=4.6,3.5 \mathrm{~Hz}), 7.19$ (t, $\left.1 \mathrm{H}, \mathrm{ArH}, J=6.9 \mathrm{~Hz}\right)$, $7.25-7.31(\mathrm{~m}, 4 \mathrm{H}, \mathrm{ThH}-3, \mathrm{H}-5, \mathrm{ArH}), 7.61$ (t, 2H, ArH, J=6.9 Hz), 7.94 (s, $1 \mathrm{H}, \mathrm{PyrH}), 13.25$ (s, 1H, COOH, $\mathrm{D}_{2} \mathrm{O}$ exchangeable). MS [EI]: m/e 328, 327, $326\left(\mathrm{M}^{+}, 10,28,100 \%\right), 325$ (30), 252 (46), 178 (32), 165 (24), 115 (37), 91 (65), 77 (31). Anal. Calcd for $\mathrm{C}_{17} \mathrm{H}_{14} \mathrm{~N}_{2} \mathrm{O}_{3} \mathrm{~S} \cdot \mathrm{H}_{2} \mathrm{O}$ : C, 59.29; H, 4.68; N, 8.13. Found: C, 59.03; H, 4.39; N, 8.31.

2-(5-Benzyl-6-oxo-3-(pyridin-3-yl)pyridazin-1(6H)-yl)acetic Acid (3d): The ester $2 \mathbf{d}(1 \mathrm{mmol})$ was dissolved in $5 \mathrm{~N} \mathrm{HCl}(20 \mathrm{ml})$. The reaction mixture was refluxed for $6 \mathrm{~h}$. The water was removed in vacuo. This compound was dried under vacuo, to obtain a hygroscopic white powder, $0.28 \mathrm{~g}$ $(87.2 \%), \operatorname{mp~} 225-226{ }^{\circ} \mathrm{C} ;{ }^{1} \mathrm{H}-\mathrm{NMR}$ (DMSO- $\left.d_{6}\right) \delta: 4.07\left(\mathrm{~s}, 2 \mathrm{H}, \mathrm{CH}_{2}\right), 4.88$ $\left(\mathrm{s}, 2 \mathrm{H}, \mathrm{CH}_{2}\right), 7.20-7.46(\mathrm{~m}, 4 \mathrm{H}, \mathrm{PyrH}, \mathrm{ArH}), 7.63(\mathrm{~d}, 2 \mathrm{H}, \mathrm{ArH}, J=7.6 \mathrm{~Hz})$, 7.86-7.92 (m, 1H, PyH-5), 8.01-8.08 (m, 2H, PyH-4, PyH-2), 8.53-8.62 (m, 1H, PyH-6), 12.9 (br s, $1 \mathrm{H}, \mathrm{COOH}, \mathrm{D}_{2} \mathrm{O}$ exchangeable).

General Procedure for the Reaction of 2-(5-Benzyl-6-oxo-3-arylpyridazin-1(6H)-yl)acetic Acid with L-Amino Acid Esters (5a-i) A mixture of 2-(5-benzyl-6-oxo-3-arylpyridazin-1(6H)-yl)acetic acid $\mathbf{3}$ (1 mmol), HATU $(0.38 \mathrm{~g}, 1 \mathrm{mmol})$ and triethylamine $\left(\mathrm{Et}_{3} \mathrm{~N}\right)(0.28 \mathrm{ml}, 2 \mathrm{mmol})$ was stirred at $0{ }^{\circ} \mathrm{C}$ for $3 \mathrm{~min}$ in DMF $(2 \mathrm{ml})$ and then amino acid $t$-butyl ester 4 $(1 \mathrm{mmol})$ was added. The reaction mixture was stirred at $0^{\circ} \mathrm{C}$ for $1 \mathrm{~h}$ and left overnight at room temperature. In the case of L-leucine $t$-butyl ester, L-glutamic di-t-butyl ester and L-aspartic di-t-butyl ester the reaction mixture was diluted with ethyl acetate $(80 \mathrm{ml})$, and the mixture was washed with $5 \%$ aqueous citric acid solution $(2 \times 10 \mathrm{ml})$, saturated sodium bicarbonate solution $(2 \times 10 \mathrm{ml})$ and saturated sodium chloride solution $(2 \times 10 \mathrm{ml})$. The organic layer was dried over anhydrous sodium sulphate, filtered and the solvent was removed in vacuo. The crude product was purified with a silica gel column using ethyl acetate/hexane $(1: 2)$ as the eluant. In the case of $\mathrm{L}_{-}(\mathrm{O}-\mathrm{t}$ $\mathrm{Bu}$ )-tyrosine-t-butyl ester and L-phenylalanine-t-butyl ester, the reaction mixture was poured into ice water, filtered, washed with $5 \%$ aqueous citric acid solution, saturated sodium bicarbonate solution, and water, and then dried and recrystallized.
tert-Butyl 2-(2-(5-Benzyl-6-oxo-3-phenylpyridazin-1(6H)-yl)acetamido)3-(4-tert-butoxyphenyl)propanoate (5a): The crude compound was recryrstallized from methylene chloride/hexane and the product was obtained as a white powder, $0.52 \mathrm{~g}(87.4 \%), \mathrm{mp} 75-76^{\circ} \mathrm{C}$; IR $(\mathrm{KBr}) \mathrm{cm}^{-1}: 3313$ ( $\mathrm{NH}), 1732$ ( $\mathrm{C}=\mathrm{O}$ ester $), 1691(\mathrm{C}=\mathrm{O}$ amide $), 1659(\mathrm{C}=\mathrm{O}$ amide $) ;{ }^{1} \mathrm{H}-\mathrm{NMR}$ $\left(\mathrm{CDCl}_{3}\right) \delta: 1.26,1.34\left(2 \mathrm{~s}, 18 \mathrm{H}, 6 \mathrm{CH}_{3}\right), 3.06\left(\mathrm{~s}, 2 \mathrm{H}, \mathrm{CH}_{2}\right), 3.97\left(\mathrm{~s}, 2 \mathrm{H}, \mathrm{CH}_{2}\right)$, $4.73(\mathrm{~m}, 1 \mathrm{H}, \mathrm{CH}), 4.95\left(\mathrm{~m}, 2 \mathrm{H}, \mathrm{CH}_{2}\right), 6.72\left(\mathrm{brd}, 1 \mathrm{H}, \mathrm{NH}, \mathrm{D}_{2} \mathrm{O}\right.$ exchangeable), $6.76(\mathrm{~d}, 2 \mathrm{H}, \mathrm{ArH}, J=8.2 \mathrm{~Hz}), 6.97(\mathrm{~d}, 2 \mathrm{H}, \mathrm{ArH}, J=8.2 \mathrm{~Hz}), 7.25$ $7.39(\mathrm{~m}, 9 \mathrm{H}, \mathrm{PyrH}, \mathrm{ArH}), 7.65(\mathrm{~m}, 2 \mathrm{H}, \mathrm{ArH})$. Anal. Calcd for $\mathrm{C}_{36} \mathrm{H}_{41} \mathrm{~N}_{3} \mathrm{O}_{5}$ : C, 72.58; H, 6.94; N, 7.05. Found: C, 72.43; H, 6.75; N, 6.89.

tert-Butyl 2-(2-(5-Benzyl-6-oxo-3-phenylpyridazin-1(6H)-yl)acetamido)3-phenylpropanoate (5b): The crude compound was recryrstallized from methylene chloride/hexane and the product was obtained as a white powder, $0.47 \mathrm{~g}(89.9 \%), \mathrm{mp} 95-96^{\circ} \mathrm{C}$; IR $(\mathrm{KBr}) \mathrm{cm}^{-1}: 3310(\mathrm{NH}), 1731(\mathrm{C}=\mathrm{O}$ ester $), 1690(\mathrm{C}=\mathrm{O}$ amide $), 1659(\mathrm{C}=\mathrm{O}$ amide $) ;{ }^{1} \mathrm{H}-\mathrm{NMR}\left(\mathrm{CDCl}_{3}\right) \delta: 1.38$ $\left(\mathrm{s}, 9 \mathrm{H}, 3 \mathrm{CH}_{3}\right), 3.10\left(\mathrm{~m}, 2 \mathrm{H}, \mathrm{CH}_{2}\right), 3.97\left(\mathrm{~s}, 2 \mathrm{H}, \mathrm{CH}_{2}\right), 4.87(\mathrm{~m}, 1 \mathrm{H}, \mathrm{CH})$, $4.87,4.97\left(2 \mathrm{~d}, 2 \mathrm{H}, \mathrm{CH}_{2}, J=14.9 \mathrm{~Hz}\right), 6.73\left(\mathrm{brd}, 1 \mathrm{H}, \mathrm{NH}, \mathrm{D}_{2} \mathrm{O}\right.$ exchangeable), 7.09, 7.14, 7.27, $7.38(4 \mathrm{~m}, 14 \mathrm{H}, \mathrm{PyrH}, \mathrm{ArH}), 7.63(\mathrm{~m}, 2 \mathrm{H}, \mathrm{ArH}) ;{ }^{13} \mathrm{C}-$ NMR $\left(\mathrm{CDCl}_{3}\right) \delta: 28.03,36.30,37.87,53.82,56.17,82.59,126.28,126.93$ $127.15,127.74,128.33,128.92,129.03,129.61,129.65,134.77,136.07$, $136.96,143.87,145.37,160.58,166.36,170.12$. MS [EI]: m/e 319 $\left(\mathrm{M}^{+}-\mathrm{C}_{14} \mathrm{H}_{20} \mathrm{O}, 2 \%\right), 303$ (18), 275 (13), 91 (28), 57 (100). Anal. Calcd for $\mathrm{C}_{32} \mathrm{H}_{33} \mathrm{~N}_{3} \mathrm{O}_{4}$ : C, 73.40; H, 6.35; N, 8.02. Found: C, 73.20; H, 6.64; N, 8.16.

tert-Butyl 2-(2-(5-Benzyl-6-oxo-3-phenylpyridazin-1(6H)-yl)acetamido)4-methylpentanoate $(\mathbf{5 c})$ : The crude compound was purified by column chromatography (ethylacetate/hexane, $1: 2$ ) and the product was obtained as a pale yellow semi-solid, $0.43 \mathrm{~g}(87.9 \%)$; IR $(\mathrm{KBr}) \mathrm{cm}^{-1}: 3305(\mathrm{NH}), 1732$ $(\mathrm{C}=\mathrm{O}$ ester $), 1690(\mathrm{C}=\mathrm{O}$ amide $), 1661(\mathrm{C}=\mathrm{O}$ amide $) ;{ }^{1} \mathrm{H}-\mathrm{NMR}\left(\mathrm{CDCl}_{3}\right) \delta$ : $0.89,0.90\left(2 \mathrm{~d}, 6 \mathrm{H}, 2 \mathrm{CH}_{3}, J=6.2 \mathrm{~Hz}\right), 1.42\left(\mathrm{~s}, 9 \mathrm{H}, 3 \mathrm{CH}_{3}\right), 1.52(\mathrm{~m}, 1 \mathrm{H}, \mathrm{CH})$ $1.63\left(\mathrm{~m}, 2 \mathrm{H}, \mathrm{CH}_{2}\right), 3.98\left(\mathrm{~s}, 2 \mathrm{H}, \mathrm{CH}_{2}\right), 4.53(\mathrm{~m}, \mathrm{H}, \mathrm{CH}), 4.95$ (br s, $\left.2 \mathrm{H}, \mathrm{CH}_{2}\right)$, 6.67 (brd, 1H, NH, D O exchangeable), 7.26-7.38 (m, 9H, PyrH, ArH), $7.65(\mathrm{~m}, 2 \mathrm{H}, \mathrm{ArH}) ;{ }^{13} \mathrm{C}-\mathrm{NMR}\left(\mathrm{CDCl}_{3} / \mathrm{DEPT}\right) \delta: 22.33,22.83\left(2 \mathrm{CH}_{3}\right), 25.01$ $(\mathrm{CH}), 28.05\left(3 \mathrm{CH}_{3}\right), 36.33,41.98\left(2 \mathrm{CH}_{2}\right), 51.73(\mathrm{CH}), 56.24\left(\mathrm{CH}_{2}\right), 82.08$ (C), 126.25, 127.11, 127.79, 128.90, 128.99, $129.57(10 \mathrm{CH}), 134.80$ $137.02,143.79,145.32(4 \mathrm{C}), 160.65,166.44,171.87(3 \mathrm{C}=\mathrm{O})$. Anal. Calcd for $\mathrm{C}_{29} \mathrm{H}_{35} \mathrm{~N}_{3} \mathrm{O}_{4}$ : C, 71.14; H, 7.21; N, 8.58. Found: C, 70.90; H, 6.97; N, 8.74 .

Di-tert-butyl 2-(2-(5-Benzyl-6-oxo-3-phenylpyridazin-1(6H)-yl)acetamido)pentanedioate $(\mathbf{5 d})$ : The crude compound was purified by column chromatography (ethylacetate/hexane, $1: 2$ ) and the product was obtained as a pale yellow semi-solid, $0.45 \mathrm{~g}(80.2 \%)$; IR $(\mathrm{KBr}) \mathrm{cm}^{-1}: 3310(\mathrm{NH}), 1735$ $(\mathrm{C}=\mathrm{O}$, ester $), 1731(\mathrm{C}=\mathrm{O}$ ester $), 1690(\mathrm{C}=\mathrm{O}$ amide $), 1660(\mathrm{C}=\mathrm{O}$ amide $)$; ${ }^{1} \mathrm{H}-\mathrm{NMR}\left(\mathrm{CDCl}_{3}\right) \delta: 1.41,1.43\left(2 \mathrm{~s}, 18 \mathrm{H}, 6 \mathrm{CH}_{3}\right), 1.92,2.11\left(2 \mathrm{~m}, 2 \mathrm{H}, \mathrm{CH}_{2}\right)$, $2.21-2.35\left(\mathrm{~m}, 2 \mathrm{H}, \mathrm{CH}_{2}\right), 3.98\left(\mathrm{~s}, 2 \mathrm{H}, \mathrm{CH}_{2}\right), 4.52(\mathrm{~m}, \mathrm{H}, \mathrm{CH}), 4.90,4.98$ $\left(2 \mathrm{~d}, 2 \mathrm{H}, \mathrm{CH}_{2}, J=14.9 \mathrm{~Hz}\right), 6.92$ (br d, $1 \mathrm{H}, \mathrm{NH}, \mathrm{D}_{2} \mathrm{O}$ exchangeable), 7.26 $7.36(\mathrm{~m}, 9 \mathrm{H}, \mathrm{PyrH}, \mathrm{ArH}), 7.64(\mathrm{~m}, 2 \mathrm{H}, \mathrm{ArH}) . \mathrm{MS}[\mathrm{EI}]: m / e 561\left(\mathrm{M}^{+}, 2 \%\right)$, $505\left(\mathrm{M}^{+}-56,2\right), 303$ (100), 275 (34), 115 (13), 91 (12), 57 (94). Anal. Calcd for $\mathrm{C}_{32} \mathrm{H}_{39} \mathrm{~N}_{3} \mathrm{O}_{6}$ : C, 68.43; H, 7.00; N, 7.48. Found: C, 68.58; H, 7.20; N, 7.36 .

Di-tert-butyl 2-(2-(5-Benzyl-6-oxo-3-phenylpyridazin-1(6H)-yl)acetamido)succinate $(\mathbf{5 e})$ : The crude compound was purified by column chromatography (ethylacetate/hexane, $1: 2$ ) and the product was obtained as a pale yellow semi-solid, $0.41 \mathrm{~g}(75.0 \%)$ yield; IR $(\mathrm{KBr}) \mathrm{cm}^{-1}: 3310(\mathrm{NH})$, $1737(\mathrm{C}=\mathrm{O}$ ester), 1731 ( $\mathrm{C}=\mathrm{O}$ ester $), 1691(\mathrm{C}=\mathrm{O}$ amide $), 1659(\mathrm{C}=\mathrm{O}$ amide); ${ }^{1} \mathrm{H}-\mathrm{NMR}\left(\mathrm{CDCl}_{3}\right) \delta: 1.40,1.43\left(2 \mathrm{~s}, 18 \mathrm{H}, 6 \mathrm{CH}_{3}\right), 2.78,2.87(2 \mathrm{dd}$, $\left.2 \mathrm{H}, \mathrm{CH}_{2}, J=17.2,4 \mathrm{~Hz}\right), 3.98\left(\mathrm{~s}, 2 \mathrm{H}, \mathrm{CH}_{2}\right), 4.71(\mathrm{~m}, \mathrm{H}, \mathrm{CH}), 4.96(\mathrm{~m}, 2 \mathrm{H}$, $\mathrm{CH}_{2}$ ), 7.07 (brd, 1H, NH, D $\mathrm{O}$ exchangeable), 7.26, $7.36(2 \mathrm{~m}, 9 \mathrm{H}, \mathrm{PyrH}$, $\mathrm{ArH}), 7.64$ (m, 2H, ArH); ${ }^{13} \mathrm{C}-\mathrm{NMR}\left(\mathrm{CDCl}_{3}\right) \delta: 27.97,28.10,36.26,37.44$ $49.40,55.86,81.63,82.47,126.31,127.09,127.75,128.85,128.99,129.48$, $129.63,134.89,137.07,143.84,145.31,160.55,166.49,169.51,170.28 . \mathrm{MS}$ [EI]: $m / e ~ 547\left(\mathrm{M}^{+}, 3 \%\right), 435$ (19), 418 (11), 303 (82), 276 (19), 102 (10), 91 (22), 96 (14), 64 (100), 57 (27). Anal. Calcd for $\mathrm{C}_{31} \mathrm{H}_{37} \mathrm{~N}_{3} \mathrm{O}_{6}: \mathrm{C}, 67.99 ; \mathrm{H}$, $6.81 ;$ N, 7.67. Found: C, 68.10; H, 7.08; N, 7.42.

tert-Butyl 2-(2-(5-Benzyl-3-(4-chlorophenyl)-6-oxopyridazin-1(6H)yl)acetamido)-3-(4-tert-butoxyphenyl)propanoate (5f): The crude compound was recryrstallized from methylene chloride/hexane and the product was obtained as a white powder, $0.54 \mathrm{~g}(85.7 \%), \mathrm{mp} 58-59^{\circ} \mathrm{C}$; IR $(\mathrm{KBr}) \mathrm{cm}^{-1}$. $3311(\mathrm{NH}), 1731(\mathrm{C}=\mathrm{O}$, ester $), 1690(\mathrm{C}=\mathrm{O}$, amide $), 1659(\mathrm{C}=\mathrm{O}$, amide); ${ }^{1} \mathrm{H}-\mathrm{NMR}\left(\mathrm{CDCl}_{3}\right) \delta: 1.28,1.36\left(2 \mathrm{~s}, 18 \mathrm{H}, 6 \mathrm{CH}_{3}\right), 3.05\left(\mathrm{~m}, 2 \mathrm{H}, \mathrm{CH}_{2}\right), 3.95$ $\left(\mathrm{m}, 2 \mathrm{H}, \mathrm{CH}_{2}\right), 4.72(\mathrm{dd}, 1 \mathrm{H}, \mathrm{CH}, J=13.0,6.1 \mathrm{~Hz}), 4.86,4.96\left(2 \mathrm{~d}, 2 \mathrm{H}, \mathrm{CH}_{2}\right.$, $J=14.6 \mathrm{~Hz}$ ), 6.69 (brd, 1H, NH, D O exchangeable), 6.77 (d, 2H, ArH, $J=8.4 \mathrm{~Hz}), 6.98(\mathrm{~d}, 2 \mathrm{H}, \mathrm{ArH}, J=8.4 \mathrm{~Hz}), 7.19(\mathrm{~s}, 1 \mathrm{H}, \mathrm{PyrH}), 7.28,7.36(2 \mathrm{~m}$, $7 \mathrm{H}, \mathrm{ArH}), 7.58$ (d, 2H, ArH, $J=8.4 \mathrm{~Hz})$. MS [EI]: $m / e 573\left(\mathrm{M}^{+}-56,4 \%\right)$, 337 (9), 107 (18), 57 (100). Anal. Calcd for $\mathrm{C}_{36} \mathrm{H}_{40} \mathrm{ClN}_{3} \mathrm{O}_{5}$ : C, 68.61; H, 
6.40; N, 6.67. Found: C, 68.36; H, 6.22; N, 6.79.

tert-Butyl 2-(2-(5-Benzyl-3-(4-chlorophenyl)-6-oxopyridazin-1(6H)yl)acetamido)-3-phenyl-propanoate $\mathbf{( 5 g})$ : The crude compound was recyrstallized from methylene chloride/hexane and the product was obtained as a white powder, $0.53 \mathrm{~g}(95.0 \%), \mathrm{mp} 54-55^{\circ} \mathrm{C}$; IR $(\mathrm{KBr}) \mathrm{cm}^{-1}: 3312$ $(\mathrm{NH}), 1730(\mathrm{C}=\mathrm{O}$ ester $), 1691(\mathrm{C}=\mathrm{O}$ amide $), 1660(\mathrm{C}=\mathrm{O}$ amide $) ;{ }^{1} \mathrm{H}-\mathrm{NMR}$ $\left(\mathrm{CDCl}_{3}\right) \delta: 1.39\left(\mathrm{~s}, 9 \mathrm{H}, 3 \mathrm{CH}_{3}\right), 3.10\left(\mathrm{~m}, 2 \mathrm{H}, \mathrm{CH}_{2}\right), 3.96\left(\mathrm{~s}, 2 \mathrm{H}, \mathrm{CH}_{2}\right), 4.75$ (dd, $1 \mathrm{H}, \mathrm{CH}, J=12.6,6.9 \mathrm{~Hz}), 4.85,4.94\left(2 \mathrm{~d}, 2 \mathrm{H}, \mathrm{CH}_{2}, J=15.3 \mathrm{~Hz}\right), 6.68$ (brd, $1 \mathrm{H}, \mathrm{NH}, \mathrm{D}_{2} \mathrm{O}$ exchangeable), $7.08(\mathrm{~s}, 1 \mathrm{H}, \mathrm{PyrH}), 7.09-7.38(\mathrm{~d}, 3 \mathrm{~m}$, $12 \mathrm{H}, \mathrm{ArH}), 7.57(\mathrm{~d}, 2 \mathrm{H}, \mathrm{ArH}, J=8.4 \mathrm{~Hz})$. MS [EI]: m/e $557\left(\mathrm{M}^{+}, 8 \%\right), 501$ $\left(\mathrm{M}^{+}-56,5\right), 337$ (39), 309 (28), 297 (12), 115 (18), 57 (100). Anal. Calcd for $\mathrm{C}_{32} \mathrm{H}_{32} \mathrm{ClN}_{3} \mathrm{O}_{4} \cdot \mathrm{H}_{2} \mathrm{O}: \mathrm{C}, 66.72 ; \mathrm{H}, 5.95 ; \mathrm{N}, 7.29$. Found: C, 66.44; $\mathrm{H}$, $5.69 ; \mathrm{N}, 7.52$

tert-Butyl 2-(2-(5-Benzyl-6-oxo-3-(thiophen-2-yl)pyridazin-1(6H)-yl)acetamido)-3-(4-tert-butoxyphenyl)propanoate $(\mathbf{5 h})$ : The crude compound was recryrstallized from methylene chloride/hexane and the product was obtained as a white powder, $0.56 \mathrm{~g}(93.2 \%), \mathrm{mp} 49-50{ }^{\circ} \mathrm{C}$; IR $(\mathrm{KBr}) \mathrm{cm}^{-1}$ : $3313(\mathrm{NH}), 1731(\mathrm{C}=\mathrm{O}$ ester $), 1691(\mathrm{C}=\mathrm{O}$ amide $), 1660(\mathrm{C}=\mathrm{O}$ amide $) ;{ }^{1} \mathrm{H}-$ NMR $\left(\mathrm{CDCl}_{3}\right) \delta: 1.27,1.35\left(2 \mathrm{~s}, 18 \mathrm{H}, 6 \mathrm{CH}_{3}\right), 3.05\left(\mathrm{~m}, 2 \mathrm{H}, \mathrm{CH}_{2}\right), 3.95(\mathrm{~m}$ $\left.2 \mathrm{H}, \mathrm{CH}_{2}\right), 4.72(\mathrm{dd}, 1 \mathrm{H}, \mathrm{CH}, J=13.0,6.9 \mathrm{~Hz}), 4.82,4.92\left(2 \mathrm{~d}, 2 \mathrm{H}, \mathrm{CH}_{2}\right.$, $J=15.3 \mathrm{~Hz}), 6.67\left(\mathrm{brd}, 1 \mathrm{H}, \mathrm{NH}, \mathrm{D}_{2} \mathrm{O}\right.$ exchangeable), $6.79(\mathrm{~d}, 2 \mathrm{H}, \mathrm{ArH}$ $J=7.7 \mathrm{~Hz}$ ), 7.00 (brd, 3H, ArH), 7.15 (s, $1 \mathrm{H}, \mathrm{PyrH}), 7.20$ (d, 1H, ThH-3, $J=3.9 \mathrm{~Hz}), 7.28,7.35(2 \mathrm{~m}, 6 \mathrm{H}, \mathrm{ThH}-4, \mathrm{H}-5, \mathrm{ArH}) . \mathrm{MS}$ [EI]: $m / e$ $\left(\mathrm{M}^{+}-55,3 \%\right), 545\left(\mathrm{M}^{+}-56,2\right), 326$ (14), 309 (26), 281 (14), 107 (19), 91 (11), 57 (100). Anal. Calcd for $\mathrm{C}_{34} \mathrm{H}_{39} \mathrm{~N}_{3} \mathrm{O}_{5} \mathrm{~S}$ : C, 67.86; H, 6.53; N, 6.98 Found: $\mathrm{C}, 68.04 ; \mathrm{H}, 6.76 ; \mathrm{N}, 6.81$.

tert-Butyl 2-(2-(5-Benzyl-6-oxo-3-(thiophen-2-yl)pyridazin-1(6H)-yl)acetamido)-3-phenyl propanoate (5i): The crude compound was recyrstallized from methylene chloride/hexane and the product was obtained as a white powder, $0.45 \mathrm{~g}(85.1 \%), \mathrm{mp} 57-58^{\circ} \mathrm{C}$; IR $(\mathrm{KBr}) \mathrm{cm}^{-1}: 3313(\mathrm{NH}), 1731$ $(\mathrm{C}=\mathrm{O}$ ester $), 1690(\mathrm{C}=\mathrm{O}$ amide $), 1659(\mathrm{C}=\mathrm{O}$ amide $) ;{ }^{1} \mathrm{H}-\mathrm{NMR}\left(\mathrm{CDCl}_{3}\right) \delta$ $1.38\left(\mathrm{~s}, 9 \mathrm{H}, 3 \mathrm{CH}_{3}\right), 3.09\left(\mathrm{~m}, 2 \mathrm{H}, \mathrm{CH}_{2}\right), 3.94\left(\mathrm{~s}, 2 \mathrm{H}, \mathrm{CH}_{2}\right), 4.75(\mathrm{dd}, 1 \mathrm{H}, \mathrm{CH}$, $J=13.0,6.1 \mathrm{~Hz}), 4.82,4.91\left(2 \mathrm{~d}, 2 \mathrm{H}, \mathrm{CH}_{2}, J=14.5 \mathrm{~Hz}\right), 6.63($ br d, $1 \mathrm{H}, \mathrm{NH}$, $\mathrm{D}_{2} \mathrm{O}$ exchangeable), $7.01(\mathrm{dd}, 1 \mathrm{H}, \mathrm{ThH}-4, J=5.4,3.9 \mathrm{~Hz}), 7.09(\mathrm{~s}, 1 \mathrm{H}$, PyrH), 7.14-7.17, 7.20, 7.26-7.38 (m, d, m, 12H, ThH-3, H-5, ArH). MS [EI]: m/e 474 (M+ $\left.\mathrm{M}^{+} 55,2 \%\right), 309$ (11), 281 (10), 91 (26), 57 (100). Anal. Calcd for $\mathrm{C}_{30} \mathrm{H}_{31} \mathrm{~N}_{3} \mathrm{O}_{4} \mathrm{~S}: \mathrm{C}, 68.03 ; \mathrm{H}, 5.90 ; \mathrm{N}, 7.93$. Found: C, 68.24; H, $6.15 ; \mathrm{N}, 8.02$

Biology The newly synthesized compounds $2, \mathbf{3}, \mathbf{5}$ were evaluated for their MAO-A inhibitory activity in vitro by the method described by Undenfriend et al. ${ }^{13)}$ using serotonin (5HT) as substrate. The method depends on determination of MAO-A activity of rat liver mitochondria. ${ }^{14)}$ All compounds under test were used at a final concentration of $1 \times 10^{-4} \mathrm{M}$. The results are expressed as a percentage inhibition of the activity of MAO-A (Table 1).

Furthermore, they were tested to determine their activity toward MAO-A and MAO-B selectivity in the presence of the specific substrate, serotonin or benzylamine, respectively. Bovine brain mitochondria were isolated according to Basford. ${ }^{15}$ The activity of MAO-A and MAO-B was determined using a fluorimetric method described by Matsumoto et al. ${ }^{16)}$ The mitochondrial fractions were preincubated at $38^{\circ} \mathrm{C}$ for $30 \mathrm{~min}$ before adding the specific inhibitor, L-deprenyl $(10.5 \mu \mathrm{M})$ to determine MAO-A activity and clorgyline $(10.5 \mu \mathrm{M})$ to determine MAO-B activity. The incubation mixture contained $(0.1 \mathrm{ml}, 0.25 \mathrm{M})$ phosphate buffer $\mathrm{pH} 7.4$, mitochondrial suspension $(6 \mathrm{mg} / 1 \mathrm{ml})$, the specific substrate for MAO-A or MAO-B $(0.1 \mathrm{~mm})$ and test compounds at four different concentrations ranging from $5 \mu \mathrm{M}$ to $0.1 \mathrm{~mm}$ dissolved in propylene glycol. The mixture was incubated in a shaking water-bath at $37^{\circ} \mathrm{C}$ for $60 \mathrm{~min}$. The reaction was quenched by adding perchloric acid. The samples were centrifuged at $10000 \mathrm{~g}$ for $5 \mathrm{~min}$ and the supernatant was completed to $2.7 \mathrm{ml}$ using $1 \mathrm{~N} \mathrm{NaOH}$ and measured with a Perkin-Elmer Lf 45 Spectrofluorimeter. Protein concentration was determined according to a previously reported method. ${ }^{17)}$ The MAO-A and MAO$\mathrm{B}$ results are expressed as $\mathrm{IC}_{50}$ (Table 1).

The test compounds were further evaluated for their oral acute toxicity in male mice using a literature method. ${ }^{19,20)}$ The results indicated that test compounds proved to be non-toxic and well tolerated by the experimental ani- mals up to $250 \mathrm{mg} / \mathrm{kg}$, although no mortality was recorded at $500 \mathrm{mg} / \mathrm{kg}$. Moreover, these compounds were tested for their parenteral toxicity. ${ }^{21)}$ The results revealed that all the test compounds were non-toxic up to $125 \mathrm{mg} / \mathrm{kg}$. We could conclude that the synthesis and biochemical evaluation of the newly synthesized pyridazines led to the design of a novel class of MAO-A inhibitors with good safety margins.

Modeling Studies Computer-assisted simulated docking experiments were carried out under MMFF94X in human MAO-A crystal structure (PDB ID: 2BXR). Docking simulation study of the synthesized compounds (2a 3a, 5d).

1) Enzyme structures were checked for missing atoms, bonds and contacts.

2) Hydrogen atoms were added to the enzyme structure. Water molecules and bound ligands were manually deleted.

3) The ligand molecules were constructed using the builder module and were energy minimized.

4) The active site was generated using the MOE-Alpha Site Finder

5) Dummy atoms were created from the obtained alpha spheres.

6) Ligands were docked within the MAO-A active site using the MOEDock with simulated annealing used as the search protocol and MMFF94X molecular mechanics forcefield for 8000 iterations.

7) The lowest energy conformation was selected and subjected to an energy minimization using MMFF94X forcefield.

\section{References and Notes}

1) Part 19 of the series: Synthetic Reactions and Structural Studies of Heterocycles Containing Nitrogen, for part 18 see ref. 8.

2) Kalgutkar A. S., Dalvie D. K., Castagnoli N., Taylor T. J., Chem. Res. Toxicol., 14, 1139-1162 (2001).

3) Bach A. W. C., Ian N. C., Johnson D. L., Abell C. W., Bembenek M. E., Kwan S. W., Seeburg P. H., Shih J. C., Proc. Nat.l Acad. Sci. U.S.A., 85, 4934-4938 (1988)

4) Fowler C. J., Tipton K. F., J. Pharm. Pharmacol., 36, 111-115 (1984).

5) Pacher P., Kohegyi E., Kecskemeti V., Furst S., Curr. Med. Chem., 8, 89-100 (2001).

6) Tetrud J. W., Koller W. C., Neurology, 63, S2-S6 (2004).

7) Riederer P., Danielczyk W., Grunblatt E., Neurotoxicology, 25, 271277 (2004).

8) Hassan S. Y., Khattab S. N., Bekhit A. A., Amer A., Bioorg. Med. Chem. Lett., 16, 1753-1756 (2006).

9) Vallejos G., Rezende M. C., Cassels B. K., J. Comput.-Aided Mol. Des., 16, 95-103 (2002)

10) Di Santo R., Costi R., Roux A., Artico M., Befani O., Meninno T., Agostinelli E., Palmegiani P., Turini P., Girilli R., Ferretti R., Gallinella B., La Torre F., J. Med. Chem., 48, 4220- 4223 (2005).

11) Chimenti F., Secci D., Bolasco A., Chimenti P., Granese A., Befani O., Turini P., Alcaro S., Ortuso F., Bioorg. Med. Chem. Lett., 14, 36973703 (2004).

12) Tao G., Irie Y., Li D.-J., Keung W. M., Bioorg. Med. Chem., 13, 4777-4788 (2005).

13) Undenfriend S., Weissbach H., Clark C. T., J. Biol. Chem., 215, 337344 (1955)

14) Schneider W. C., J. Biol. Chem., 176, 259-266 (1948).

15) BasfordR. E., Methods Enzymol., 10, 96-101 (1967).

16) Matsumoto T., Suzuki O., Furuta T., Asai M., Kurokawa Y., Rimura Y., Katsumata Y., Takahashi I., Clin. Biochem., 18, 126-129 (1985).

17) Bradford M. M., Anal. Biochem., 72, 248-254 (1976).

18) Verma M., Tripathi M., Saxena A. K., Shanker K., Eur. J. Med. Chem., 29, 941-946 (1994).

19) Litchfield J. T., Wilcoxon F., J. Pharmacol. Exp. Ther, 96, 99-113 (1949).

20) Bekhit A. A., Fahmy H. T. Y., Arch. Pharm. Pharm. Med. Chem., 336, $111-118$ (2003).

21) El Massry A. M., Amer A., Heterocycles, 29, 1907-1914 (1989).

22) Jahine H., Zaher H. A., Sayed A., Seada M., Indian J. Chem., 15B $352-355(1977)$ 